\title{
Glycyrrhizin therapy for viral infections
}

\author{
Kei Numazaki \\ Department of Pediatrics, Sapporo Medical University, School of Medicine, Sapporo, Hokkaido, 060-8543, Japan. Tel: \\ (011) 611-2111 EXT.3413, Fax: (011)611-0352, E-mail: numazaki@sapmed.ac.jp. \\ Accepted 26 September 2003

\begin{abstract}
Glycyrrhizin (GL) was reported as the most active in inhibiting replication of the severe acute respiratory syndrome (SARS)-associated coronavirus. Therapeutic effect of GL for liver dysfunction associated with cytomegalovirus (CMV) infection in immunocompetent individuals was evaluated. Liver dysfunction in 4 cases improved and CMV disappeared from urinary samples after administration of GL intravenously by the age of 12 months. GL treatment also should be applied for the patients with SARS.
\end{abstract}

Key words: Glycyrrhizin, SARS, cytomegalovirus.

It was reported that glycyrrhizin (GL) is the most active in inhibiting replication of the SARS-associated coronavirus and GL should be assessed for treatment (Cinatl et al., 2003). Some antiviral agents that express activity against virus-specific metabolic processes without displaying cytotoxicity have become available. Unfortunately, until present time, no successful treatment of SARS has yet been completely developed. GL, a Chinese herbal drug extracted from licorice roots, has shown to express antiviral effects and immunomodulating activities. Cell-free infection of HIV-1 and HIV-2 is almost completely blocked in the presence of GL. Cell-to-cell infection by HIV-1, HIV-2, and human T-cell lymphotropic virus type $I$ is also inhibited by $G L$.

Although the exact mechanism of antiviral effects of GL is unclear, some therapeutic and prophylactic effects of GL on chronic active viral hepatitis have been claimed in Japan. It has been reported that suppressor $\mathrm{T}$ cells or macrophages generated by stimulation with allogenic lymphocytes were inhibited by intravenous administration of GL (Miyaji et al., 2002). It is likely that expression of viral genome on $\mathrm{T}$ lymphocytes as well as activities of some cytokines are associated with active viral infection. GL has been demonstrated to exhibit interferon-inducing and NK-enhancing activities.

Human cytomegalovirus (CMV) is the most common cause of congenital and perinatal infections throughout the world. We had evaluated anti-CMV properties GL both in vivo and in vitro (Numazaki and Chiba, 1993; Numazaki et al., 1994; Numazaki, 1998). Clinical symptoms and laboratory abnormal findings improved and CMV disappeared sooner from clinical specimens after intravenous administration of $\mathrm{GL}$ than in control.

In previous study, therapeutic effect of $G L$ for liver dysfunction associated with CMV infection in immunocompetent individuals was evaluated (Numazaki, 1998). I administered $0.2 \% \mathrm{GL}$ dissolved in saline (2 $\mathrm{mg} / \mathrm{ml} \mathrm{GL}$ ), supplemented with $2 \%$ glycine and $0.1 \%$ cysteine (Stronger Neo-Minophagen C, SNMC) intravenously to 4 infants (GL was administered $10-20 \mathrm{mg}$ $/ \mathrm{kg} /$ day intravenously for 8 weeks). I also administrated Glycyron (25 mg GL supplemented with $25 \mathrm{mg}$ methionine and $25 \mathrm{mg}$ glycine) orally to another 6 infants (GL was administered 2-4 mg/kg/day orally for 12 weeks). 
Liver dysfunction in 4 cases improved and CMV disappeared from urinary samples after administration of GL intravenously by the age of 12 months. In 6 infants liver dysfunction normalized and CMV disappeared by the age of 16 months after administration of GL orally. CMV disappeared from urine sooner after intravenous administration of $G L$ than in controls $(p<0.05)$. No side effects were noted during the treatment with $G L$. GL therapy was considered to be a suitable treatment for improving liver dysfunction in immunocompetent infants and children associated with CMV infection.

GL treatment also should be applied for the patients with SARS. At first, GL treatment for SARS should be restricted for a short time. Unusual side effects such as hypertension and hypokalaemia were reported in some patients after several months of GL treatment. However, taken along with infrequent and very mild side effects, long-term intermittent GL treatment also would benefit patients with chronic phase of SARS by maintaining their quality of life with easier compliance.

\section{REFERENCES}

Cinatl J, Morgenstern B, Bauer G, Chandra P, Rabenau H, Doerr HW (2003). Glycyrrhizin, an active component of liquorice roots, and replication of SARS-associated coronavirus. Lancet 361: 2045-2046.

Miyaji C, Miyakawa R, Watanabe H, Kawamura H, Abo T (2002). Mechanisms underlying the activation of cytotoxic function mediated by hepatic lymphocytes following the administration of glycyrrhizin. Int. Immunopharmacol. 2: 1079-1086.

Numazaki K, Chiba S (1993). Natural course and trial of treatment for infantile liver dysfunction associated with cytomegalovirus infections. In Vivo 7: 477-480.

Numazaki K, Nagata N, Sato T, Chiba S (1994). Effect of glycyrrhizin, cyclosporin $A$, and tumor necrosis factor on infection of U-937and MRC- 5 cells by human cytomegalovirus. J. Leukocyte Biol. 55: 24-28.

Numazaki K, Umetsu M, Chiba S (1994). Effect of glycyrrhizin in children with liver dysfunction associated with cytomegalovirus infection. Tohoku J. Exp. Med. 172: 147-153.

Numazaki K (1998). Glycyrrhizin therapy for liver dysfunction associated with cytomegalovirus infection in immunocompetent children. Antimicrobics and Infectious Diseases Newsletter 117: 70-71. 